\title{
The Influence of Grip Width on Training Volume During the Bench Press with Different Movement Tempos
}

\author{
by \\ Michal Wilk1, Mariola Gepfert ${ }^{1}$, Michal Krzysztofik ${ }^{1}$, Artur Golas ${ }^{1}$, \\ Aleksandra Mostowik' Adam Maszczyk², Adam Zajac ${ }^{1}$
}

The aim of the study was to determine the effect of the wide-grip bench press (WGBP) and the close-grip bench press (CGBP) on the number of performed repetitions (REPs) and time under tension (TUT) using a variable tempo of movement. Twenty (20) women experienced in resistance training were enrolled in the study (1RM-CGBP $=55.2 \pm 9.5$ $\mathrm{kg} ; 1 R M-W G B P=52.7 \pm 8.5 \mathrm{~kg}$ ). Participants performed 5 sets of the BP with a maximal number of REPs at $70 \% 1 R M$. Different tempos of movement, i.e., slow (6/0/X/0) and fast (2/0/X/0), and grip widths, i.e., the CGBP and the WGBP, were employed. The following variables were registered: maximal number of repetitions in every set (REPset-5), total number of repetitions performed in 5 sets (TREP), maximal time under tension in every set (TUTset-5) and total time under tension in 5 sets (TTUT). The two-way ANOVA revealed statistically significant differences between the WGBPFAST and the WGBPSLOW in TUTset1-5 $(p<0.05)$ and TTUT $(p<0.01)$, as well as between the CGBPFAST and the CGBP sLow in TUT Set1-5 $(p<0.01)$ and TTUT $(p<0.01)$. Significant differences between the WGBPFAST and the WGBP $P_{\text {sLOW }}$ were also observed in REP Set1-5 $(p<0.01)$ and TREP $(p<0.01)$ as well as between the CGBPFAST and the CGBP sLOw in REP Set1-5 $(p<0.01)$ and TREP $(p<0.01)$. No significant differences between the WGBPsLow and the CGBPsLow nor the WGBP FAST and the CGBPFAST were found. The study demonstrates that the tempo of movement, regardless of the width grip, has a significant effect on the volume of effort in resistance training.

Key words: resistance exercise, repetition, time under tension, close-grip, wide-grip.

\section{Introduction}

The levels of strength and power are important aspects of many sport disciplines (Young et al., 2015). For the upper limbs and the torso, the bench press (BP) is one of the most often used resistance exercises in developing strength and power output (Algra, 1982; Gomo and Tillaar, 2016; Lehman, 2005). The BP is usually performed with an individually adjusted grip width, which was defined by Young et al. (2015) as "the strongest position". However, athletes may also use different grip widths to vary their training routines. These may include the wide-grip bench press (WGBP) or the close-grip bench press (CGBP). The WGBP is described as a bench press with a grip width of $200 \%$ or more of the bi-acromial distance (BAD), while the CGBP is defined as a grip width of $95 \%$ BAD (Barnett et al., 1995; Lockie et al., 2017a, 2017b; Wagner et al., 1992). The CGBP and WGBP have been frequently used in training methodology and scientific research. Studies have indicated that the grip width used in the CGBP may be better suited for specific movements used in many sports (Delextrat and Cohen, 2009; Stokes et al., 2010; Wheeler and Sayers, 2011). However, Wagner et al. (1992) found that the WGBP (165 - 200\% BAD) allowed for a higher maximum external load compared to the CGBP. The 6RM loads were $11.1 \%$ greater using a WGBP compared to the CGBP (Saeterbakken et al., 2017). When comparing the CGBP to the WGBP, one should notice that in the

1 - Department of Sports Training, Jerzy Kukuczka Academy of Physical Education in Katowice, Poland.

2 - Department of Statistics and Methodology, Jerzy Kukuczka Academy of Physical Education in Katowice, Poland.

Authors submitted their contribution to the article to the editorial board.

Accepted for printing in the Journal of Human Kinetics vol. 68/2019 in August 2019. 
second variation of the exercise the bar starts and finishes in a position that is closer to the chest, thus reducing the distance the bar must travel (Madsen and McLaughlin, 1984).

Although the BP is arguably the most popular upper body exercise, few studies have evaluated the effect of different grip widths on the generated muscle force (Wagner et al., 1992) or power output (Baker et al., 2001; Stock et al., 2010). Furthermore, studies by Gomo and Tillaar (2016) and Lockie et al. (2017a, 2017b) have shown that a change in grip width has a significant impact on the generated power, force and velocity of movement. Contrary to those findings, Lockie et al. (2018) did not find significant changes in the level of power output for different external loads (\%RM) between the traditional BP and the CGBP. Differences in muscle strength and power output between different barbell grip widths in the BP (Lockie et al., 2017a), may be partly related to changes in muscle activity (Saeterbakken et al., 2017). For example, increasing the grip width to $200 \%$ BAD leads to a higher activation of the pectoralis major muscle (Barnett et al., 1995; Clemons and Aaron 1997; Lehman, 2005) and the biceps brachii muscle (Lehman, 2005), while simultaneously decreasing the activity of the anterior part of the deltoid muscle (Barnett et al., 1995) and the triceps brachii muscle compared to the 100\% BAD width (Barnett et al., 1995; Lehman, 2005; Maszczyk et al., 2016).

Although some previous studies have demonstrated significant differences in strength and power generated depending on the width of the grip used during the BP (Lockie et al., 2017a, $2017 \mathrm{~b}$ ), none of them referred to different movement tempos during the BP exercise. The movement tempo is usually described using a sequence of digits (e.g. 2/0/X/0), where each digit determines the duration (s) of a particular phase of the movement. Since there is no standardized method of assigning these digits within the scientific literature, in this paper we adopted a unified description of the movement tempo as follows: eccentric / pause / concentric / pause. Change in the movement tempo during a strength exercise impacts on the value of exercise volume, the level of generated force, power output, and hypertrophy (Hatfield et al., 2006; Headley et al., 2011; Sakamoto and Sinclair, 2006; Wilk et al., 2018a).
The number of repetitions performed at a specific tempo influences the total time under tension in a set (TUT) as well as total time under tension (TTUT). Time under tension is an indicator of the work performed by the muscles in both the ECC and CON phases of the movement regardless of the number of performed REPs (Wilk et al., 2019a, 2019b). In a study of experienced resistance training athletes, Wilk et al. (2018a) compared the slow (6/0/4/0), medium (5/0/3/0) and fast (2/0/2/0) movement tempos during the bench press exercise at $70 \% 1 \mathrm{RM}$. Despite the fact that all sets were performed until muscle failure, values for TTUT and the total number of performed repetitions (TREP) were significantly different between particular tempos. The highest values for TTUT after 5 sets of the bench press were observed at the slow movement tempo $(178.8 \pm 33.69 \mathrm{~s})$, whereas significantly lower values were found for the medium (166.60 $\pm 29.27 \mathrm{~s})$ and fast (124.65 \pm 33.66 s) tempos. On the other hand, TREP was significantly higher in the faster tempo (28.32 \pm 6.86) compared to the medium $(18.75 \pm 4.14)$ and the slow (15.71 \pm 4.03$)$ tempo. Burd et al. (2012) also compared the effects of slow (6/0/6/0) and fast $(1 / 0 / 1 / 0)$ movement tempos on TTUT, but with an equal number of REPs performed in a set, and using the same loads. They found that TTUT was significantly higher for a slower $(407 \pm 23$ s) compared to a fast $(50 \pm 3 \mathrm{~s})$ movement tempo. This leads to the conclusion that the movement tempo has a significant influence not only on the number of repetitions performed, but also time under tension.

Although the BP is the most common exercise of the upper body, no scientific studies have analysed the effect of grip width relative to a controlled movement tempo. Therefore, the aim of the study was to determine the effect of different grip widths (WGBP vs. CGBP) on exercise volume determined by the number of REPs and TUT using a variable tempo of movement in a group of resistance trained females. We hypothesize that different tempos of movement and different grip widths used in the BP would induce significant differences in TTUT and TREP values in resistance trained females.

\section{Methods}

Experimental Approach to the Problem

The experiment was performed following 
a randomized crossover design, where each participant performed a familiarization session with a 1RM test and four different testing protocols 3-4 days apart. Participants performed the WGBP with a 2/0/X/0 (WGBPFAst), and a $6 / 0 / X / 0$ (WGBPsLow) tempo, respectively. They also went through the same exercise procedures with the CGBP using a 2/0/X/0 (CGBPFAST) and a 6/0/X/0 (CGBPslow) tempo. During each experimental session, participants performed five sets using $70 \% 1 \mathrm{RM}$ with $3 \mathrm{~min}$ rest intervals in between. The maximal number of repetitions was performed in each set. The following variables were registered: the maximal number of repetitions in every set (REPset-5), the total number of repetitions performed in 5 sets (TREP), maximal time under tension in every set (TUTset1-5) and total time under tension in 5 sets (TTUT). Participants were required to refrain from resistance training 48 hours prior to each experimental session, were familiarized with the protocol as well as the benefits and potential risks of the study, and provided their written consent for participation in the study.

Participants

Twenty (20) healthy female athletes, experienced in resistance training $(3.9 \pm 0.63 \mathrm{yrs})$ volunteered for the study after completing an ethical consent form (age $=27.3 \pm 2.2$ years; body mass $=53.3 \pm 7.7 \mathrm{~kg} ;$ CGBP $1 \mathrm{RM}=55.2 \pm 9.5 \mathrm{~kg}$; WGBP $1 \mathrm{RM}=52.7 \pm 8.5 \mathrm{~kg}$; mean $\pm \mathrm{SD}$ ). The inclusion criterion was to be able to perform a bench press with the load of at least $100 \%$ of the body mass. Participants were allowed to withdraw from the experiment at any moment and were free from any musculoskeletaldisorders. The study protocol was approved by the Bioethics Committee for Scientific Research, at the Academy of Physical Education in Katowice, Poland, and performed according to the ethical standards of the Declaration of Helsinki, 1983.

Procedures 1RM WGBP and CGBP Strength Testing

All testing was performed in the Strength and Power Laboratory at the Academy of Physical Education in Katowice. Participants arrived at the laboratory between 9:00 and 11:00 a.m. and cycled on an ergometer for 5 minutes, which followed by a general upper body warm-up of 10 pull ups and 15 push-ups. Next, participants performed 15, 10, and $5 \mathrm{BP}$ repetitions using $20 \%, 40 \%$, and $50 \%$ of their estimated 1RM, respectively. The maximum strength test with the WGBP was performed first. To this end, participants lay on a flat bench with their feet on the floor, and their head, shoulders and buttocks resting flat on the bench. The grip width adopted for the WGBP was 200\% BAD (Maszczyk et al., 2014; Saeterbakken et al., 2017; Wagner et al., 1992). The grip width was marked on the barbell with athletic tape, and a pronated grip was used. Participants placed their hands on the bar at the same position for each set. Then they executed single repetitions using a volitional cadence with a 3-min rest interval between successive trials. The load for each subsequent attempt was increased by $2.5 \mathrm{~kg}$, and the process was repeated until failure. No more than five attempts were allowed before the 1RM was determined for each participant. After a $10 \mathrm{~min}$ recovery period, participants completed the maximum strength test in the CGBP (Lockie et al., 2017a, 2017b). The body position and constraints that determined a successful lift were the same as those for the WGBP, except for the different grip width. The grip width adopted for the CGBP was 95\% BAD (Lockie et al., 2017a, 2017b; Wagner et al., 1992), with hands placed in a position within the shoulders. The grip width was marked on the barbell with athletic tape, and a pronated grip was used as in the WGBP. Following the established procedures, the warm-up for the second strength test began by completing 3-5 repetitions at $85 \%$ of the participants' estimated 1RM, and then one repetition with 90\%1RM. Afterwards, participants attempted their first 1RM lift following a 3-min recovery period, and this process continued until $1 R M$ was reached. For both the WGBP and the CGBP, absolute strength was taken as the maximum load lifted. An IPF Eleiko bar and weight plates (Eleiko, Sport AB Sweden) were used for both the WGBP and the CGBP.

Experimental sessions

The general and specific warm-up before the experimental sessions was identical to that used during the familiarization session. After the warm up, participants performed 5 consecutive sets of the bench press with particular grip width (WGBP or CGBP) and a specified tempo (2/0/X/0 or $6 / 0 / \mathrm{X} / 0$ ) with $70 \% 1 \mathrm{RM}$ following a metronome guided movement cadence in the eccentric phase (Korg MA-30, Korg, Melville, New York, USA). The concentric phase was performed at the 
maximal tempo of movement. Each experimental set was performed to concentric failure. All repetitions were performed without bouncing the barbell off the chest, without intentionally pausing at the transition between the eccentric and concentric phases, and without raising the lower back off the bench. The intervals between experimental sessions were 3-4 days. All familiarization and experimental sessions were recorded by means of a Sony camera (FDR191 AX53). Time under tension and the number of performed repetitions were obtained manually from the recorded data using slow speed playback ( $1 / 5$ speed). In order to ensure the reliability of manual data collection, four independent researchers analyzed the data from the Sony camera (Wilk et al., 2019b). There were no significant differences in the TUT (s), TTUT (s) and REP (n), and TREP (n) between the data collected by 4 evaluators. All participants completed the described testing protocol.

The following variables were registered independently for the WGBPFAST, WGBPsLow, CGBPFAST, and CGBPsLow:

- $\mathrm{TUT}_{(\mathrm{set}-5)}(\mathrm{s})$ - time under tension for each set (1 to 5$)$

- $\quad$ TTUT (s) - total time under tension for 5 sets

- $\operatorname{REP}_{(\mathrm{Set1}-5)}$ (n) - number of repetitions performed for each set (1 to 5)

- TREP (n) - total number of repetitions performed for 5 sets

\section{Statistical analysis}

Time under tension, the number of repetitions, total time under tension, and the total number of repetitions were expressed as mean \pm SD. Before using the parametric test, the assumption of normality was verified using the Kolmogorov-Smirnov test. Two-way ANOVA was used to show differences between study variables. The level of significance was set at $p<0.05$. When appropriate, the Bonferroni post hoc test was used to compare selected data. The remaining analyses were performed using STATISTICA software (StatSoft, Inc., Tulsa OK Oklahoma, USA, 2018 version 12).

\section{Results}

There were significant differences between WGBP Fast and WGBPsLow variables in TUTset1-5 $(p<$ $0.05)$ and TTUT $(p<0.01)$, as presented in Table 1. Similarly, there were significant differences between CGBPFast and CGBPslow variables in TUTSet1-5 $(p<0.01)$ and TTUT $(p<0.01)$ (Table 1$)$. There were no significant differences between WGBPFAST and CGBPFAST variables in TUTset1-5 and in TTUT, nor between the WGBPsLow and the CGBPsLow.

There were significant differences between WGBPFAST and WGBPsLow variables in $\operatorname{REP}_{\text {Set } 1-5}(p<$ $0.005)$ and TREP $(p<0.005)$ (Table 2). Additionally, there were significant differences between CGBPfast and CGBPslow variables in $\operatorname{REP}_{\text {set1-5 }}(p<$ $0.01)$ and total TREP $(p<0.005)$ (Table 2$)$.

\begin{tabular}{|c|c|c|c|c|c|c|}
\hline \multirow{4}{*}{ Set } & \multicolumn{3}{|c|}{$\begin{array}{c}\text { Table } \mathbf{1} \\
\text { under tension using a variable tempo of movement and different grip widths. }\end{array}$} & \multicolumn{3}{|c|}{ Table 1} \\
\hline & \multicolumn{6}{|c|}{ Time Under Tension (s) } \\
\hline & \multicolumn{3}{|c|}{ Close-grip bench press } & \multicolumn{3}{|c|}{ Wide-grip bench press } \\
\hline & $2 / 0 / \mathrm{X} / 0$ & $6 / 0 / \mathrm{X} / 0$ & $p$ & $2 / 0 / \mathrm{X} / 0$ & $6 / 0 / X / 0$ & $p$ \\
\hline$B P_{\text {Set } 1}$ & $43.8 \pm 12.3$ & $60.3 \pm 20.1$ & 0.001 & $49.9 \pm 12.9$ & $64.3 \pm 10.7$ & 0.003 \\
\hline$B P_{\text {Set } 2}$ & $37.5 \pm 9$ & $52.6 \pm 15.3$ & 0.001 & $40.9 \pm 6.5$ & $53.6 \pm 11.5$ & 0.001 \\
\hline$B P_{\text {Set3 }}$ & $31.7 \pm 8.6$ & $31.7 \pm 8.6$ & 0.001 & $33.8 \pm 7.1$ & $44.5 \pm 10.6$ & 0.001 \\
\hline$B P_{\text {Set }}$ & $29.5 \pm 7.8$ & $46.8 \pm 16.2$ & 0.001 & $30.6 \pm 9.3$ & $39.3 \pm 8.8$ & 0.027 \\
\hline$B P_{\text {Set } 5}$ & $26.6 \pm 8.9$ & $38.8 \pm 11.2$ & 0.001 & $26.8 \pm 8.3$ & $32.3 \pm 12.6$ & 0.027 \\
\hline Totalset $1-5$ & $169.1 \pm 43.3$ & $230.3 \pm 63.9$ & 0.001 & $182.1 \pm 39.6$ & $234.3 \pm 48.0$ & 0.002 \\
\hline \multicolumn{7}{|c|}{ data presented as mean \pm standard deviation (SD) } \\
\hline
\end{tabular}




\begin{tabular}{|c|c|c|c|c|c|c|}
\hline \multicolumn{7}{|c|}{$\begin{array}{c}\text { Table } 2 \\
\text { Number of performed repetitions using a variable tempo of movement and different grip widths. }\end{array}$} \\
\hline \multirow{3}{*}{ Set } & \multicolumn{6}{|c|}{ Number of repetitions (n) } \\
\hline & \multicolumn{3}{|c|}{ Close-grip bench press } & \multicolumn{3}{|c|}{ Wide-grip bench press } \\
\hline & $2 / 0 / X / 0$ & $6 / 0 / \mathrm{X} / 0$ & $p$ & $2 / 0 / X / 0$ & $6 / 0 / X / 0$ & $p$ \\
\hline$B P_{S e t 1}$ & $11.2 \pm 3.1$ & $9.5 \pm 2$ & 0.001 & $12.7 \pm 4.2$ & $10.6 \pm 2.4$ & 0.003 \\
\hline$B P_{\text {Set2 }}$ & $9.5 \pm 3$ & $7.8 \pm 1.9$ & 0.005 & $10.5 \pm 3.3$ & $8.9 \pm 2.6$ & 0.001 \\
\hline$B P_{\text {Set3 }}$ & $8.1 \pm 2.8$ & $6.6 \pm 2.2$ & 0.001 & $8.9 \pm 3.1$ & $7.5 \pm 2.5$ & 0.001 \\
\hline$B P_{\text {Set } 4}$ & $7 \pm 2.6$ & $5.8 \pm 1.8$ & 0.001 & $7.9 \pm 3.3$ & $6.5 \pm 2.4$ & 0.004 \\
\hline$B P_{\text {Set5 }}$ & $5.7 \pm 2.7$ & $4.6 \pm 1.8$ & 0.001 & $6.5 \pm 3$ & $5.3 \pm 2.6$ & 0.002 \\
\hline Totalset1-5 & $41.5 \pm 12.9$ & $34.3 \pm 8.9$ & 0.002 & $46.5 \pm 15.9$ & $38.8 \pm 11.2$ & 0.002 \\
\hline \multicolumn{7}{|c|}{ data presented as mean \pm standard deviation (SD) } \\
\hline
\end{tabular}

\section{Discussion}

The main finding of the study was that the movement tempo had a significant effect on the number of performed REPset1-5, TREP as well as TUTset1-5 and TTUT. The significant differences in

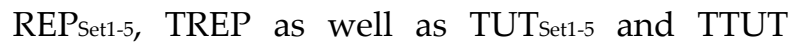
between the slow and the fast tempo of movement occurred for both the CGBP and the WGBP, which partially confirms our hypothesis. Importantly, the study did not show differences between the WGBP and the CGBP in maximal volume of exercise (REP and TUT), which indicates that grip width in the BP does not affect exercise volume when using different tempos of movement.

To date, studies have confirmed the effect of the movement tempo on exercise volume, both in terms of the number of performed REPs (Sakamoto and Sinclair, 2006; Wilk et al., 2018a, 2018b), and TUT (Hunter et al., 2003; Tran et al., 2006; Wilk et al., 2018a; 2018b). Wilk et al. (2018a) showed that changes in the movement tempos $(2 / 0 / 2 / 0 ; 5 / 0 / 3 / 0 ; 6 / 0 / 4 / 0)$ had a significant effect on the maximum number of performed REPs and TUT during the WGBP. Sakamoto and Sinclair (2006) confirmed the effect of the movement tempo on the number of REPs performed in the shoulder press and the squat. However, based on the results, it was not possible to determine precisely whether those changes resulted from modification of the duration of the CON phase, the ECC phase, or both phases at the same time. The current study showed that both the CGBP and the WGBP performed at $6 / 0 / \mathrm{X} / 0$ led to a significant increase in TUTset1-5 as well as TTUT compared to the $2 / 0 / \mathrm{X} / 0$ tempo of movement. On the contrary, the REPset1-5 as well as TREP were significantly higher at $2 / 0 / X / 0$ compared to $6 / 0 / X / 0$, regardless of the grip width.

The study showed that changes in the duration of the ECC phase only significantly affected REPset1-5, TREP as well as TUTset1-5 and TTUT, regardless of the grip width used. In our study, the duration of the ECC phase in the slow tempo was three times longer compared to the fast tempo of movement (ECCSLOW - $6 \mathrm{~s}$; ECCFAST $2 \mathrm{~s}$ ), and, as found by Lastayo et al. (1999), the ECC phase requires up to 6-7 times less oxygen compared to the CON phase of the movement. Additionally, studies have shown that the CON phase contributes most to the $\mathrm{O} 2$ uptake of lifting and recovery, with the ECC phase adding less to O2 uptake (Dudley et al., 1991; Seliger et al., 1968). Results presented by Seliger et al. (1968), Dudley et al. (1991), and Lastayo et al. (1999), provide the basis for the physiological explanation of higher 
TUT as well TTUT at the $6 / 0 / \mathrm{X} / 0$ compared to the 2/0X/0 tempo of movement. Contrary to the results of Sakamoto and Sinclair (2006) and Wilk et al. (2018a), which showed that changes in REP and TUT were caused by the duration of both the CON and ECC phases, it was clearly demonstrated that significant changes in TUT and REP could occur through modification of the duration of the ECC phase only. Thus, longer duration of the ECC phase of the movement increases exercise time, which consequently increases TUT, what can be particularly important in the development of hypertrophy and also anaerobic endurance in different sport disciplines.

Contrary to the TUTset1-5 and TTUT, the highest number of TREP was recorded under the fast tempo of the movement $(2 / 0 / \mathrm{X} / 0)$ condition. The higher number of REPs performed in the $2 / 0 / X / 0$ tempo resulted from the duration of the ECC phases of the movement. With three times shorter duration of the ECC phase of the movement at the 2/0/X/0 tempo, the CON phase was performed three times more often in a given unit of time, which directly translated into a significantly higher number of REPset-5. The higher number of REPSet1-5 and TREP completed at the $2 / 0 / X / 0$ tempo compared to the $6 / 0 / X / 0$ was registered for both the WGBP and the CGBP. An additional factor that could lead to a higher number of REPs completed at $2 / 0 / X / 0$ was the more efficient and more frequent use of the stretchshortening cycle (SSC). The use of the SSC helps generate higher values of muscular force and power, and accelerations during the $\mathrm{CON}$ phase of the movement, compared to performing only a concentric explosive bench press (Clark et al., 2010; Cronin et al., 2001; Cronin and Henderson, 2004; Newton et al., 1997). A faster tempo of the entire movement cycle without a pause between the ECC and CON phases also impacts the increased activation and more effective utilization of the SSC (Malisoux et al., 2006). On the contrary, a decrease in the movement tempo for the $6 / 0 / \mathrm{X} / 0$ reduces the frequency and efficiency of the SSC (Cronin et al., 2001), both in the WGBP and the CGBP. The results confirm the scientific reports of Sakamoto and Sinclair (2006), as well as Wilk et al. (2018a), who demonstrated that a faster movement tempo allowed for a higher number of REPs using a specified external load. The number of REPs performed is an indicator of exercise volume in resistance training, however, if a different tempo of movement or different duration of the eccentric phase is used, the number of REPs cannot be considered a reliable indicator of training volume.

The results of the study did not show significant differences between the WGBP and the CGBP in exercise volume in a group of female athletes which is contradictory to the previously formulated hypothesis. No significant differences in TUTset1-5, REPSet1-5, TTUT and TREP between the CGBP and WGBP groups were observed, both at the $2 / 0 / X / 0$ and the $6 / 0 / X / 0$ tempo. This result is surprising since studies to date have confirmed significant differences between the CGBP and the WGBP in the level of generated muscle strength and power output, as well as muscle activity (Madsen and McLaughlin, 1984; McLaughlin, 1985). Furthermore, Saeterbakken et al. (2017) recorded significantly higher values of $1 \mathrm{RM}$ for the WGBP compared to the CGBP $(132.7 \pm 17 \mathrm{~kg}$ vs. $119.2 \pm 16.6 \mathrm{~kg}$ ), those results were also confirmed by other researchers (Lockie et al., 2017a, 2017b; Saeterbakken et al., 2017; Wagner, 1992); however, in this study no significant differences in 1RM between the CGBP and the WGBP were found. Moreover, there was a tendency for higher 1RM results in the CGBP compared to the WGBP. The lack of significant differences in 1RM between the WGBP and the CGBP in the study may be the reason for the lack of significant differences in exercise volume. The differences between the CGBP and the WGBP described in previous studies have concerned not only the 1RM level, but also differences resulting from movement biomechanics (Clemons and Aaron, 1997; Gomo and Tillar, 2016; Lehman, 2005). However, biomechanical differences between the WGBP and the CGBP did not cause significant differences in exercise volume, determined by both the REP and TUT values. Lack of significant differences in REPSet1-5 and TUTset1-5 as well as TREP and TTUT values between the WGBP and the CGBP indicates that there is no need for independent determination of exercise volume when using different grip widths in the BP. The limitation of the present study pertains to the grip width used as participants declared that the BP grip used in their previous training was closer to the CGBP rather than the WGBP. It can be stated thus that to some extent, the WGBP was a 'new technique' to them; however, we think that it did not affect the 
obtained results.

\section{Conclusions}

The present study demonstrates that changes of the movement tempo, and more accurately, changes of the duration of the ECC phase, have a significant effect on the number of performed REPs as well as TREP, TUT, and TTUT in resistance training. Furthermore, the research results suggest that time under tension can be an additional indicator of the effort, which should be monitored and taken into consideration during periodization of training programs. Therefore, there is a need for further analysis of movement velocities in particular resistance exercises, using identical resistance training protocols in order to more accurately examine the effects of the movement tempo.

\section{Acknowledgements}

The study was supported and funded by the statutory research of the Jerzy Kukuczka Academy of Physical Education in Katowice, Poland - research leaders Michal Wilk, as well as by a grant of the Ministry of Science and Higher Education in Poland NRSA4 04054.

\section{References}

Algra B. In-depth analysis of the bench press. Nat Strength Cond Assoc J, 1982; 4: 6-13

Baker D, Nance S, Moore M. The load that maximizes the average mechanical power output during explosive bench press throws in highly trained athletes. J Strength Cond Res, 2001; 15: 20-24

Barnett C, Kippers V, Turner P. Effects of variations of the bench press exercise on the EMG activity of five shoulder muscles. J Strength Cond Res, 1995; 9: 222-227

Burd NA, Andrews RJ, West DW, Little JP, Cochran AJ, Hecktor AJ, Cashback G, Gibala MJ, Potvin JR, Baker SK, Philips SM. Muscle time under tension during resistance exercise stimulates differential muscle protein sub-fractional synthetic responses in men. J Physiol, 2012; 2: 351-361

Clemons JM, Aaron C. Effect of grip width on the myoelectric activity of the prime movers in the bench press. J Strength Cond Res, 1997; 11: 82-87

Clark R, Bryant A, Hao Pua Y. Examining different aspects of functional performance using a variety of bench throw techniques. J Strength Cond Res, 2010; 24: 2755-2761

Cronin JB, McNair PJ, Marshall RN. Magnitude and decay of stretch-induced enhancement of power output. Eur J Appl Physiol, 2001; 84: 575-581

Cronin JB, Henderson M. Maximal strength and power assessment in novice weight training. J Strength Cond Res, 2004; 18: 48-52

Delextrat A, Cohen D. Strength, power, speed, and agility of women basketball players according to playing position. J Strength Cond Res, 2009; 23: 1974-1981

Dudley GA, Tesch PA, Harris MS, Golden CL, Buchanan P. Influence of eccentric actions on the metabolic cost of resistance exercise. Aviat Space Environ Med, 1991; 62: 678-682

Gomo O, Van Den Tillaar R. The effects of grip width on sticking region in bench press. J Sports Sci, 2016; 34: 232-238

Hatfield DL, Kraemer WJ, Spiering BA, Häkkinen K, Volek JS, Shimano T, Spreuwenberg LP, Silvestre R, Vingren JL, Fragala MS, Gómez AL, Fleck SJ, Newton RU, Maresh CM. The impact of velocity of movement on performance factors in resistance exercise. J Strength Cond Res, 2006; 20: 760-766

Headley SA, Henry K, Nindl BC, Thompson BA, Kraemer WJ, Jones MT. Effects of lifting tempo on one repetition maximum and hormonal responses to a bench press protocol. J Strength Cond Res, 2011; 25: 406-413 
Hunter GR, Seelhorst D, Snyder S. Comparison of metabolic and heart rate responses to super slow vs. traditional resistance training. J Strength Cond Res, 2003; 17: 76-81

Lastayo PC, Reich TE, Urquhart M, Hoppeler H, Lindstedt SL. Chronic eccentric exercise: improvements in muscle strength can occur with little demand for oxygen. Am J Physiol, 1999; 276: 611-615

Lehman GJ. The influence of grip width and forearm pronation/supination on upper-body myoelectric activity during the flat bench press. J Strength Cond Res, 2005; 19: 587-591

Lockie RG, Callaghan SJ, Moreno MR, Risso FG, Liu TM, Stage AA, Birmingham-Babauta SA, Stokes JJ, Giuliano DV, Lazar A, Davis DL, Orjalo AJ. An investigation of the mechanics and sticking region of a one-repetition maximum close-grip bench press versus the traditional bench press. Sports (Basel), 2017a; 5(3): 46

Lockie RG, Callaghan SJ, Moreno MR, Risso FG, Liu TM, Stage AA, Birmingham-Babauta SA, Stokes JJ, Giuliano DV, Lazar A, Davis DL, Orjalo A. Relationships between mechanical variables in the traditional and close-grip bench press. J Hum Kinet, 2017b; 60: 19-28

Lockie RG, Callaghan SJ, Orjalo AJ, Moreno MR. Loading range for the development of peak power in the close-grip bench press versus the traditional bench press. Sports, 2018; 6: 97

Madsen N, McLaughlin T. Kinematic factors influencing performance and injury risk in the bench press exercise. Med Sci Sports Exerc, 1984; 16: 376-381

Malisoux L, Francaux M, Nielens H, Theisen D. Stretch-shortening cycle exercises: an effective training paradigm to enhance power output of human single muscle fibers. J Appl Physiol, 2006; 100: 771-779

Maszczyk A, Gołaś A, Pietraszewski P, Roczniok R, Zając A, Stanula A. Application of Neural and Regression Models in Sports Results Prediction. Procedia - Soci Behavio Sci, 2014; 117: 482-487

Maszczyk A, Golas A, Czuba M, Krol H, Wilk M, Stastny P, Goodwin J, Kostrzewa M, Zajac A. EMG analysis and modelling of flat bench press using artificial neural networks. SAJRSPER, 2016; 38: 91-103

McLaughlin TM. Grip spacing and arm position. Powerlifting USA, 1985; 8: 24

Newton RU, Murphy AJ, Humphries BJ, Wilson GJ, Kraemer WJ, Häkkinen HA. Influence of load and stretch shortening cycle on the kinematics, kinetics and muscle activation that occurs during explosive upperbody movements. Eur J Appl Physiol Occup Physiol, 1997; 75: 333-342

Saeterbakken AH, Mo DA, Scott S, Andersen V. The effects of bench press variations in competitive athletes on muscle activity and performance. J Hum Kinet, 2017; 57: 61-71

Sakamoto A, Sinclair PJ. Effect of movement velocity on the relationship between training load and the number of repetitions of bench press. J Strength Cond Res, 2006; 20: 523-527

Seliger V, Dolejs L, Karas V, Pachlopnikova I. Adaptation of trained athletes' energy expenditure to repeated concentric and eccentric muscle contractions. Eur J Appl Physiol, 1968; 26: 227-234

Stock MS, Beck TW, Defreitas JM, Dillon MA. Relationships among peak power output, peak bar velocity, and mechanomyographic amplitude during the free-weight bench press exercise. J Sports Sci, 2010; 28: 13091317

Stokes JV, Luiselli JK, Reed DD, Fleming RK. Behavioral coaching to improve offensive line pass-blocking skills of high school football athletes. J Appl Behav Anal, 2010; 43: 463-472

Tran QT, Docherty D, Behm D. The effects of varying time under tension and volume load on acute neuromuscular responses. Eur J Appl Physiol, 2006; 98: 402-410

Wagner LL, Evans SA, Weir JP, Housh TJ, Johnson GO. The effect of grip width on bench press performance. Int J Sport Biomech, 1992; 8: 1-10

Wheeler KW, Sayers MGL. Rugby union contact skills alter evasive agility performance during attacking ball carries. Int J Sports Sci Coach, 2011; 6: 419-432 
Wilk M, Golas A, Krzysztofik M, Zajac A. The effects of eccentric cadence on power and velocity of the bar during the concentric phase of the bench press movement. J Sports Sci Med, 2019a; 18: 191-197

Wilk M, Krzysztofik M, Maszczyk A, Chycki J, Zajac A. The acute effects of caffeine intake on time under tension and power generated during the bench press movement. J Int Soc Sports Nutr 2019b; 16: 8

Wilk M, Golas A, Stastny P, Nawrocka M, Krzysztofik M, Zajac A. Does tempo of resistance exercise impact training volume? J Hum Kinet, 2018a; 62: 241-250

Wilk M, Stastny P, Golas A, Nawrocka M, Jelen K, Zajac A, Tufano J. Physiological responses to different neuromuscular movement task during eccentric bench press. Neuro Endocriol Lett, 2018b; 39: 101-107

Young KP, Haff GG, Newton RU, Gabbett TJ, Sheppard JM. Assessment and monitoring of ballistic and maximal upper-body strength qualities in athletes. Int J Sports Physiol Perform, 2015; 10: 232-237

\section{Corresponding author:}

\section{Michal Wilk}

Department of Sports Training, Jerzy Kukuczka Academy of Physical Education

in Katowice, Poland

ul. Mikolowska 72a,

40-065 Katowice, Poland,

E-mail: m.wilk@awf.katowice.pl 